Math. Ann. 301, 813-820 (1995)

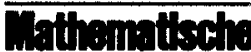

\title{
Classification of recurrent domains for some holomorphic maps
}

\section{John Erik Fornæss, Nessim Sibony}

1 Department of Mathematics, University of Michigan, Ann Arbor, MI 48109-1003, USA

2 Mathématiques, Université de Paris-Sud, Bâtiment 425, F-91405 Orsay Cedex, France

Received: 5 April 1994

Mathematics Subject Classification (1991): 32A99, 58F12

\section{Introduction}

Let $f: P^{2} \rightarrow P^{2}$ be a holomorphic map of degree $d \geqq 2$ on the two dimensional projective space. Then $f$ is given in homogeneous coordinates by $\left[f_{0}: f_{1}: f_{2}\right]$ where each $f_{j}$ is a homogeneous polynomial of degree $d$ and the $f_{j}$ have no common zero except the origin. Observe that $f$ is a $d^{2}$ to one map. We denote by $H_{d}$ the family of such self maps. In analogy with the one variable theory the Fatou set of $f$ is the maximal open set where the family $\left(f^{n}\right)$ is equicontinuous. A Fatou component is a connected component of the Fatou set. The higher dimensional analog of the Fatou Julia theory has been studied in [FS1] [FS2]. We will always assume that $d \geqq 2$.

Our purpose in this paper is to study periodic Fatou components $\Omega$ for $f$, so $f^{k}(\Omega)=\Omega$ for some $k \geqq 1$. Without lack of generality we can assume that $f(\Omega)=\Omega$ replacing $f$ by an iterate if necessary.

Definition 0.1. A Fatou component $\Omega$ is recurrent if for some $p_{0} \in \Omega$ the $\omega$-limit set of $p_{0}$ intersects $\Omega$. More precisely there exists $p_{0} \in \Omega$ such that $f^{n_{i}}\left(p_{0}\right)$ is relatively compact in $\Omega$ for some subsequence $n_{i}$.

In $P^{1}$, the recurrent Fatou components are attractive basins, Siegel discs and Herman rings. For the one variable theory we refer to [CG] or [Be].

We describe in Theorem 1.2 the analogous components for holomorphic maps in $\mathbb{P}^{2}$.

In a second paragraph we study the recurrent Fatou components for Hénon mappings in $\mathbb{P}^{2}$. Compare with [BS].

Hénon mappings are holomorphic polynomial automorphisms of $\mathbb{C}^{2}$, they are the dynamically interesting ones, see [FM], and they do not extend as holomorphic maps from $\mathbb{P}^{2}$ to $\mathbb{P}^{2}$. 


\section{Holomorphic maps in $\mathbb{P}^{2}$}

Let $f: \mathbb{P}^{2} \rightarrow \mathbb{P}^{2}$ be a holomorphic map, $f \in H_{d}$. Assume $\Omega=f(\Omega)$ is a recurrent Fatou component. It is a priori possible that there exists $p_{0} \in \Omega$ such that the orbit $f^{n}\left(p_{0}\right)$ clusters both at some interior point and at some boundary point of $\Omega$. In $\mathbb{P}^{1}$ this cannot happen, we will show that this does not happen in $\mathbb{P}^{2}$ either. This is a trivial consequence of the classification of recurrent domains in Theorem 1.2.

If $f$ is a rational map in $\mathbb{P}^{1}$ there are only finitely many recurrent domains. A recent theorem by E. Gavosto [G] shows that holomorphic maps on $\mathbb{P}^{2}$ can have infinitely many recurrent domains.

It is shown in [FS1] that any Fatou component is a domain of holomorphy. A recent result of Ueda [U] shows that every Fatou component is Kobayashi hyperbolic.

Definition 1.1. A Fatou component $\Omega$ is a Siegel domain if there exists a subsequence $\left(f^{n_{i}}\right)$ converging uniformly on compact sets of $\Omega$ to identity.

We have the following result.

Theorem 1.2. Let $f \in H_{d}, d \geqq 2$. Let $\Omega$ be a recurrent Fatou component such that $f(\Omega)=\Omega$. Then one of the following happens:

(i) There is a fixed attractive point $p \in \Omega$, the eigenvalues $\lambda_{1}, \lambda_{2}$ of $f^{\prime}$ at $p$ satisfy $\left|\lambda_{1}\right|<1,\left|\lambda_{2}\right|<1$.

(ii) There exists a Riemann surface $\tilde{\Sigma}$ which is a closed complex submanifold of $\Omega$ and $f \mid \tilde{\Sigma} \rightarrow \tilde{\Sigma}$ is an automorphism, moreover $d\left(f^{n}(K), \tilde{\Sigma}\right) \rightarrow 0$ for any compact set $K$ in $\Omega$. The Riemann surface $\tilde{\Sigma}$ is biholomorphic to a disc, a punctured disc or an annulus and $f \mid \tilde{\Sigma}$ is conjugate to a rotation. The limit $h$ of any convergent subsequence, $f^{n_{i}}$, has the same image. Any two limits $h_{1}, h_{2}$ differ only by a rotation in $\tilde{\Sigma}$.

(iii) The domain $\Omega$ is a Siegel domain. Any limit of a convergent subsequence of $\left(f^{n}\right)$ is an automorphism of $\Omega$.

Proof. Since $\Omega$ is recurrent, there exists $p_{0} \in \Omega$ and $n_{i} \rightarrow \infty$ such that $f^{n_{i}}\left(p_{0}\right)$ is relatively compact in $\Omega$. In this case we prove that we are in one of the situations described in i), ii), iii).

Assume $f^{n_{i}}\left(p_{0}\right) \rightarrow p, n_{i+1}-n_{i} \rightarrow \infty$. Taking a subsequence $\{i=i(j)\}$ and recalling that we are in the Fatou set, we can suppose that the sequence $\left\{f^{n_{i+1}-n_{i}}\right\}_{i}$ converges uniformly on compact sets in $\Omega$ to a holomorphic map $h: \Omega \rightarrow \bar{\Omega}$. Let $p_{i}=f^{n_{i}}\left(p_{0}\right)$. Then $f^{n_{i+1}-n_{i}}\left(p_{i}\right)=f^{n_{i+1}}\left(p_{0}\right)=p_{i+1}$. Hence $f^{n_{i+1}-n_{i}}(p)=p_{i+1}+O\left(\left|p_{i}-p\right|\right)$ so converges to $p$. Therefore, necessarily $h(p)=p$.

Consider all maps $h: \Omega \rightarrow \bar{\Omega}$, with $h(p)=p$ for some $p \in \Omega$ and $h=\lim f^{k_{j}}$ for some subsequence $k_{j}$.

Let $F$ ix $(h)$ denote the collection of fixed points of $h$. Since $h$ commutes with $f$, it follows that $f$ maps $F i x(h)$ to itself.

If, for some $h$ the rank of $h$ is 0 , then $h(\Omega)=p$ and necessarily $f(p)=p$, hence $p$ is also a fixed point for $f$. Also both eigenvalues of $f^{\prime}$ at $p$ must have 
modulus strictly less than one since some iterates of $f$ converge to the constant map. Hence this leads to case i).

Assume for some $h$ the rank of $h$ is two. Then for some sequence $f^{k_{i+1}-k_{i}}$ $\rightarrow I d$ and hence $\Omega$ is a Siegel domain. The restriction of $f$ to $\Omega$ is clearly an automorphism of $\Omega$. We are then in case (iii). We want to show that if $\left(f^{n_{j}}\right)$ converge to a map $g$ then $g$ is an automorphism of $\Omega$.

Let $\operatorname{Aut}(\Omega)$ denote the holomorphic automorphisms of $\Omega$. We know from Ueda's, [U], result that $\Omega$ is Kobayashi hyperbolic, so $\operatorname{Aut}(\Omega)$ has the structure of a Lie group [Ko]. Let $\tilde{G}:=$ closed subgroup generated in $\operatorname{Aut}(\Omega)$ by $f$. Since $G$ is a closed subgroup of a Lie group it is a Lie group. Let $G^{0}$ be the connected component of $I d$ in $\tilde{G}$, it is also a Lie group. Since $\left(f^{n_{i}}\right)$ converges to identity, then $G^{0}$ is not reduced to identity. But $G^{0}$ is clearly commutative, hence it is isomorphic to $\mathbb{T}^{k} \times \mathbb{R}^{l}$. Let $\Phi$ denote the isomorphism from $\mathbb{T}^{k} \times \mathbb{R}^{l}$ to $G^{0}$. For some $(a, b) \in \mathbb{T}^{k} \times \mathbb{R}^{l}$, we have $\Phi(a, b)=f$. If $b \neq 0$ we cannot have $\left(f^{n_{i}}\right)$ converging to $I d$. So $b=0$ and hence $G^{0}$ is isomorphic to $\mathbb{T}^{k}$, consequently $G^{0}$ is compact. It follows that each convergent subsequence of $\left(f^{n}\right)$ tend to an element of $A u t(\Omega)$.

We now assume that for all $h$, the maximal rank of $h$ is one. Fix an $h$ and let $\Sigma:=h(\Omega)$. Then $\Sigma \subset \bar{\Omega}$. For $p \in \Omega$, there is an irreducible piece of a Riemann surface with singularities $\Sigma_{p} \subset \bar{\Omega}$ and a neighborhood $U(p)$ so that $h(U(p))=\Sigma_{p}$. We define an abstract Riemann surface $R$ as the union $\bigcup \Sigma_{p_{i}}$ for a covering $U\left(p_{i}\right)$ of $\Omega$, with the identifications at $q \in \Sigma_{p_{i}} \cap \Sigma_{p_{j}}$ if the two pieces agree as germs. Then $R$ is Hausdorff by the identity theorem.

The map $h: \Omega \rightarrow \Sigma$ factors naturally as a map $h=\pi \circ \bar{h}$ where $\bar{h}: \Omega \mapsto R$ and $\pi: R \mapsto \Sigma$.

We show first that $f$ is a surjective self map of $\Sigma$.

If $x \in \Sigma$ then $x=h(y)$ for some $y \in \Omega$ and $f(x)=f(h(y))=h(f(y)) \in \Sigma$. So $f(\Sigma) \subset \Sigma$. We show next that the restriction of $f$ to $\Sigma$ is surjective on $\Sigma$.

Let $x=h(y), y \in \Omega$. Choose $y_{-} \in \Omega$ such that $f\left(y_{-}\right)=y$. Define $x_{-}=h\left(y_{-}\right)$. Then $x_{-} \in \Sigma$ and $f\left(x_{-}\right)=f\left(h\left(y_{-}\right)\right)=h f\left(y_{-}\right)=h(y)=x$.

Define $\Sigma^{0}:=\Sigma \cap \Omega$. Since $f(\Omega) \subset \Omega$, then $f\left(\Sigma^{0}\right) \subset \Sigma^{0}$. Since $f$ is an open map ([FS1]), $f$ maps the boundary of $\Omega$ to itself and hence $f\left(\Sigma^{0}\right)=\Sigma^{0}$.

We first show that $h$ is not constant on the irreducible component $\Sigma_{p}$ of $\Sigma \cap \Omega$ which contains $p$. Assume not. Since $h(p)=p$, then $h \mid \Sigma_{p} \equiv p \in \Sigma$. But $f^{2 k_{i}} \rightarrow p$ so we are in case i).

Since $f^{k_{i+1}-k_{i}}\left(f^{k_{i}}\right)=f^{k_{i+1}}$ we can assume, using a diagonal process, that for a subsequence $m_{i},\left(f^{m_{i}}\right)$ converges to a new map $h$ and $h=I d$ on $\Sigma_{p}$. Since $f^{l} \circ h=h \circ f^{l}$ it follows that $h=I d$ on each $f^{l}\left(\Sigma_{p}\right), l \geqq 1$. We use this new $h$ from now on.

We want to show that $\bigcup_{1 \geqq 0} f^{l}\left(\Sigma_{p}\right)$ is closed in $\Omega$. We know that $\bigcup_{l \geqq 0} f^{\prime}\left(\Sigma_{p}\right) \subset S:=\{q ; q \in \Omega, h(q)=q\}$. Since $I d-h^{\prime}$ has at least rank one, $S$ is a countable union of disjoint irreducible components each of which is a point or a smooth complex curve. It follows that $\Sigma_{p}$ is a component of $S$ and since $f$ is a proper self map of $\Omega, \bigcup_{l \geqq 0} f^{l}\left(\Sigma_{p}\right)$ is a closed countable union of irreducible curves in $S$. 
Suppose $\Sigma$ is a torus. Then $f: \Sigma \rightarrow \Sigma$ is an $l$ to 1 map, $l \geqq 2$ by Proposition 7.5 ([FS1]). Hence repelling points for $f \mid \Sigma$ are dense in $\Sigma$, which contradicts normality in $\Omega$.

We next show that if $\Sigma$ is a $\mathbb{P}^{1}, \mathbb{C}$ or $\mathbb{C}^{*}$ then $f \mid \Sigma$ is an automorphism. Suppose not, then $f$ is an $l$ to 1 surjective $\operatorname{map} f: \Sigma \rightarrow \Sigma$ with $l \geqq 2$. From the Fatou-Julia theory in one variable repelling periodic points for $f \mid \Sigma$ are dense in the Julia set of $f \mid \Sigma$. Choose $q$ a repelling periodic point for $f \mid \Sigma$ say $f^{s}(q)=q$ and $h\left(z_{0}\right)=q$ with $z_{0} \in \Omega$. Recall that $h=\lim f^{m_{1}}$. We can assume $h^{\prime}\left(z_{0}\right) \neq 0$ in some direction.

Fix $0<\delta \ll 1$. For each $l \in$ choose $r_{l}>0$ such that $f^{l s}\left(B\left(q, r_{l}\right)\right) \subset B(q, \delta)$. Choose $m_{i(l)}$ such that $f^{m_{i}(l)}\left(z_{0}\right) \in B\left(q, r_{l}\right)$. Then $f^{i s+m_{i(1)}}\left(z_{0}\right) \in B(q, \delta)$. The sequence $\left(f^{l s+m_{l}(1)}\right)_{l}$ is equicontinuous, hence we can even assume that in a ball $B_{1}$ containing $z_{0}$, we have $f^{l s+m_{i(t)}}\left(B_{1}\right) \subset B(q, \delta)$. We can always increase $m_{i(l)}$ so that $f^{l s+m_{i(1)}}$ is as close as we wish to $f^{l s} \circ h$. Then the derivative of $f^{\left(s+m_{i(l)}\right.}$ at $z_{0}$ is not bounded in all directions, a contradiction. Hence we have shown that if $\Sigma$ is a $\mathbb{P}^{\mathbf{1}}, \mathbb{C}$ or $\mathbb{C}^{*}$ then $f \mid \Sigma$ is an automorphism. As a consequence $\Sigma$ cannot be a $P^{1}$ since by Theorem 7.5 in [FS1] $f \mid \Sigma$ cannot be an automorphism.

So if $\Sigma$ is a $\mathbb{C}$ or $\mathbb{C}^{*}$, since $\left.f^{m_{i}}\right|_{\Sigma_{p}} \rightarrow I d$, and $\bigcup f^{l}\left(\Sigma_{p}\right)$ is closed, then necessarily $f$ (or $f^{2}$ ) is conjugate to an irrational rotation. This proves our claim.

If $\Sigma$ is hyperbolic, we use the classification of holomorphic maps $g: \Sigma \rightarrow \Sigma$ in Theorem 3.3 [M]. Since $f^{n_{i}} \rightarrow I d$ on the open set $\Sigma_{p}$ of $\Sigma$, we know that not all orbits converge to an attracting fixed point, nor do all orbits diverge to infinity. From Proposition 7.5 in [FS1], we know also that $f$ is not of finite order, hence Theorem 3.3 in [M] implies that $\Sigma$ is isomorphic to the unit disc $D, D^{*}$ or an annulus and $f$ is conjugate to an irrational rotation.

We want to prove next that $\Sigma$ is independent of $h$.

Assume $f^{m_{i}} \rightarrow k$ uniformly on compact sets of $\Omega$. Let $\Sigma^{\prime}=k(\Omega)$. We have $f^{m_{i}}\left|\Sigma^{0} \rightarrow k\right| \Sigma^{0}$ but since $f$ is conjugate to a rotation on $\Sigma^{0}, k\left(\Sigma^{0}\right) \subset \Sigma^{0}$ and $\Sigma^{\prime}$ is an extension of $\Sigma^{0}$ and one can prove similarly that $f$ is conjugate to rotation on $\Sigma^{\prime}$. Similarly $\Sigma^{0}$ is an extension of $\Sigma^{\prime} \cap \Omega$, so $\Sigma^{\prime} \cap \Omega=\Sigma^{0}$. Let $\tilde{\Sigma}$ be the maximal extension of $\Sigma$ in $\bar{\Omega}$ such that $f$ is conjugate to a rotation on $\tilde{\Sigma}$. We then get that $\left(f^{n}\right)$ converges u.c.c. on $\Omega$ to $\tilde{\Sigma}$, i.e. $d\left(f^{n}, \tilde{\Sigma}\right) \rightarrow 0$. We would like to show next that $\Sigma=\Sigma^{\prime}$. Pick a point $p$ in $\Sigma^{0}$. Then we can find a holomorphic coordinate system in a neighborhood of $p$ such that in that neighborhood $\Sigma=\{w=0 ; a<|z|<b\}$ and

$$
f(z, w)=\left(e^{i \theta} z+w g_{1}(z, w), w a_{1}(z)+w^{2} k_{1}(z, w)\right) .
$$

Then

$$
f^{n}(z, w)=\left(e^{n i \theta} z+O(w), w \prod_{j=0}^{n-1} a_{1}\left(e^{i j \theta} z\right)+O\left(w^{2}\right)\right) .
$$

Write $a_{n}(z)=\prod_{j=0}^{n-1} a_{1}\left(e^{i j 0} z\right)$. Since we are in a Fatou component, the functions $a_{n}(z)$ are necessarily uniformly bounded on any smaller set 
$a^{\prime}<|z|<b^{\prime}, a<a^{\prime}, b^{\prime}<b$. We must even have that $a_{n} \rightarrow 0$ uniformly, since all limits have rank 1. It follows that a neighborhood of $\Sigma^{0}$ is attracted to $\Sigma^{0}$.

An easy estimate gives that if we start with small enough $w$, and consider tangent vectors $v=(1, \alpha)$ based at $(z, w),|\alpha|$ small enough, then $\left(f^{n}\right)^{\prime}(v)=$ $c_{n}\left(1, \alpha_{n}\right), \alpha_{n} \rightarrow 0,|| c_{n}|-1|$ as small as we want.

For each $n$, let $\mathscr{F}_{n}$ be the "vertical" foliation consisting of leaves $L$ with $f^{n}(L) \subset\{z=$ const $\}$. We show next that $\mathscr{F}_{n} \rightarrow \mathscr{F}$, a foliation with leaves of the form $z=g(w)$.

The above observation shows that in order to compute the horizontal distance between leaves of $\mathscr{F}_{n}$ and corresponding leaves of $\mathscr{F}_{n+1}$, it is enough to compute the distance after applying $f^{n}$. If one considers the leaves of $\mathscr{F}_{n}$ as almost vertical discs of radius $C$ then after applying the map $f, n$ times, the discs have radius at most $c \mathrm{Cr}^{n}$ for some $r<1$.

Since the discs of $\mathscr{F}_{1}=f^{n}\left(\mathscr{F}_{n+1}\right)$ and $\left(z_{1}=\right.$ constant $)=f^{n}\left(\mathscr{F}_{n}\right)$ start at the same point, they can be at most at $\mathrm{CCr}^{n}$ away from each other. So the horizontal distance between $\mathscr{F}_{n}$ and $\mathscr{F}_{n+1}$ is at most $\mathrm{Cr}^{n}$, so $\mathscr{F}_{n}$ converge to a foliation $\mathscr{F}$ with leaves of the form $z=g(w)$.

Moreover $f$ maps leaves of $\mathscr{F}$ to leaves of $\mathscr{F}$. It follows that these leaves are in level sets of $h$. In particular, in a neighborhood of $\Sigma \cap \Omega$, the level sets of $h$ are independent of $h$.

Next, pick any two limits $h_{1}, h_{2}$, say $h_{i}(\Omega)=\Sigma_{i}$. By connectivity reasons one must contain the other, say $\Sigma_{1} \subset \Sigma_{2}$. Let $\lambda_{\theta}$ denote rotation by $\theta$ in $\Sigma_{2}$. Then near one component of $\Sigma_{1} \cap \Omega$, there must exist a $\theta$ so that $h_{1} \equiv \lambda_{\theta} \circ h_{2}$. But then this must hold everywhere. So $\Sigma_{1}=\Sigma_{2}$. In particular, the level sets of $h_{1}$ and $h_{2}$ are the same (even globally) and $f$ maps level sets to level sets (globally). It follows that if $p_{0} \in \Omega$, then either $\left\{f^{n}\left(p_{0}\right)\right\}$ converges to the boundary or is a relatively compact set in $\Omega$.

Note that using the local coordinates above, it follows from the maximum principle that $\Sigma \cap \Omega$ cannot have more than one component. Indeed, let $A$ be a subannulus of $\Sigma$ whose boundary with respect to $\Sigma$ is in $\Omega$. Assume $A$ intersects $\partial \Omega$. Then $A$ has a Stein neighborhood isomorphic to $A \times$ disc, and we can apply the maximum principle there. Since $\left(f^{n}\right)$ converges towards $\Sigma$ near the boundary of $A$ we still have convergence in a neighborhood of $A$, so $A \subset \Omega$.

Next we prove that actually $\Sigma$ is a closed complex manifold of $\Omega$. Namely, let us assume not. We consider a circle in $\Sigma$ so that one side is in $\Omega$ and the other side is in the boundary. We then choose a local coordinate system as above. Consider the coefficient $a_{1}(z)$. For each radius $r$ let $A(r)$ denote the average of $\log \left|a_{1}(z)\right|$ over the circle of radius $r$. Similarly let $A_{n}(r)$ denote the average of $\log \left|a_{n}(z)\right|$. Then $A_{n}(r)$ and $A(r)$ have the same sign always and they are continuous and monotonic. Also note that the rotation by $\theta$ on the circle is ergodic. Hence it follows that $\frac{1}{n} \log \left|a_{n}(z)\right| \rightarrow A(r)$ in $L^{2}$ on the circle $|z|=r$. Note that the functions $\frac{1}{n} \log \left|a_{n}\right|$ are equicontinuous so they converge uniformly to $A(r)$ except near circles where $a_{1}$ has a zero. 
In particular it follows that if $A(r)<0$, then the circle with radius $r$ is in the Fatou component. Since $A(|z|)$ is subharmonic, it follows that $A(r)>0$ on the side which belongs to the boundary. But then it follows from ergodicity that for large $n,\left|a_{n}(z)\right|>1$ uniformly, on circles $|z|=r$. But this implies that these points repell points from $\Omega$. Hence there can be no points in $\Omega$ converging to them. So $\Sigma=\Sigma^{0} \subset \Omega$.

From Ueda's Theorem ([U]) $\Omega$ is Kobayashi hyperbolic so $\Sigma$ which is contained in $\Omega$ cannot be $\mathbb{C}$ or $\mathbb{C}^{*}$. This finishes the proof.

We describe more precisely the structure of the closure of iterates in the case of a Siegel domain.

Proposition 1.3. Let $f: \mathbb{P}^{2} \rightarrow \mathbb{P}^{2}, f \in H_{d}$. Let $\Omega=f(\Omega)$ be a Fatou component which is a Siegel domain. Let $G$ denote the closure of $\left(f^{n}\right)_{n \geqq 0}$ in the topology of uniform convergence on compact sets. Then $G$ is a sub-Lie group of Aut $(\Omega)$ and $G$ is isomorphic to $\mathbb{T}^{k} \times F$ where $F$ is a finite group and $k=1$ or 2 .

Proof. We have shown in the proof of Theorem 1.2 that $\tilde{G}:=$ \{closed subgroup generated by $f\} \cap A u t \Omega$ is a compact Lie group which is isomorphic to $\mathbb{T}^{k} \times F$ where $F$ is a finite group.

Since $\Omega$ is Kobayashi hyperbolic and of complex dimension two, it follows from a theorem of Kruzhilin $[\mathrm{Kr}]$ that the maximal real dimension of a commutative group in $\operatorname{Aut}(\Omega)$ is two. Indeed Kruzhilin shows that, fields that commute in the Lie algebra are real linearly independent iff they are complex linearly independent; so $0 \leqq k \leqq 2$. We cannot have $k=0$ since an iterate of $f$ cannot be the identity, see [FS1]. Assume $k=2$. Then we have an effective $\mathbb{T}^{2}$ action on $\Omega$. It follows from a theorem of Barrett, Bedford, Dadok [BBD] that there exists a hyperbolic Reinhardt domain $U$ in $\mathbb{C}^{2}$ and a biholomorphic map $\Phi: \Omega \rightarrow U$, such that for some $l, \Phi \circ f^{l}=R \circ \Phi$ where $R(z, w)=\left(e^{i \alpha} z, e^{i \beta} z\right)$. So $f$ is conjugate to $R_{1}(z, w)=\left(e^{i \alpha / l} z, e^{i \beta / l} w\right)$, hence $\tilde{G}=\mathbb{T}^{2} \times F$ and also $G=\tilde{G}$.

When $k=1$, then $\tilde{G} \simeq \mathbb{T} \times F$ where $A$ is a finite group, it is also clear in that case that $G=\bar{G}$.

\section{Examples.}

1. Let $f[z: w: t]=\left[\lambda z t+z^{2}: \lambda^{p} w t+w^{2}: t^{2}\right]$ where $\lambda=e^{2 i n \theta}, p \in \mathbb{Z}^{+}$and $\theta$ is a diophantine number. Then $f$ has a Siegel component $\Omega$ with $[0: 0: 1] \in \Omega$. Since in a neighborhood of $(0,0)$ in $\mathbb{C}^{2},(z, w) \rightarrow\left(\lambda z+z^{2}, \lambda^{p} w+w^{2}\right)$ is conjugate to $\left(\lambda z, \lambda^{p} w\right), G$ is isomorphic to $\mathbb{T}$, which can be seen as the subgroup in $\mathbb{T}^{2}$ generated by $\left(\lambda, \lambda^{p}\right)$.

2. Let $g[z: w: t]=\left[\lambda z t+z^{2}: \mu w t+w^{2}: t^{2}\right]$ with $\lambda=e^{2 i \pi \theta}, \mu=e^{2 i \pi \psi}$ with $\lambda, \mu$ satisfying the Brjuno condition, see $[\mathrm{H}]$, in order that $f$ be linearizable near $[0: 0: 1]$ then $G$ is isomorphic to $\mathbb{T}^{2}$.

\section{Hénon mappings}

In this paragraph we want to study recurrent periodic domains for Hénon mappings. 
Recall that $f: \mathbb{C}^{2} \rightarrow \mathbb{C}^{2}$ is a Hénon map if it is a finite composition of maps of the following type

$$
f_{j}(z, w)=\left(p_{j}(z)-a w, z\right)
$$

where $p_{j}$ is a polynomial of degree $d_{j} \geqq 2$. Hénon mappings are the dynamically interesting polynomial automorphisms of $\mathbb{C}^{2}$, see [FM], [BS], [FS3]. The article [FS4] is a survey.

We just recall the following facts. Let $K^{+}=\left\{(z, w), f^{n}(z, w), n \geqq 0\right.$, is bounded $\}, K^{-}=\left\{(z, w) \backslash f^{-n}(z, w), n \geqq 0\right.$ is bounded $\}$ and $K=K^{+} \cap K^{-}$. Then $U^{+}:=\mathbb{C}^{2} \backslash K^{+}$is the basin of attraction of infinity, more precisely if we identify $\mathbb{C}^{2}$ with the open set $t \neq 0$ in $\mathbb{P}^{2}$, then points in $U^{+} \subset \mathbb{P}^{2}$ converge towards $p^{+}:=[1: 0: 0]$ in $\mathbb{P}^{2}$. The sets $K^{+}$and $K^{-}$are closed. The sequence $\left(f^{n}\right)$ converge uniformly on compact sets of $K^{+}$towards $K$, which is compact.

We describe the Fatou components in the interior of $K^{+}$which are recurrent. The question was considered in [BS] and [FS3]. We however want to give more details for the case ii) in the following theorem.

Theorem 2.1. Let $f \mathbb{C}^{2}: \rightarrow \mathbb{C}^{2}$ be a Hénon mapping. Let $\Omega$ be a recurrent Fatou component in the interior of $K^{+}$. Assume $f(\Omega)=\Omega$. Then $\Omega$ is of one of the following types:

(i) There is a fixed attracting point $p \in \Omega$ and $\Omega$ is biholomorphic to $\mathbb{C}^{2}$.

(ii) There exists a Riemann surface $\tilde{\Sigma}$ which is a closed complex submanifold in $\Omega$ such that $d\left(f^{n}(X), \tilde{\Sigma}\right) \rightarrow 0$ for any compact $X$ in $\Omega$. The Riemann surface $\tilde{\Sigma}$ is biholomorphic to a disc, a punctured disc or an annulus and $f \mid \tilde{\Sigma}$ is conjugate to an irrational rotation.

(iii) The domain $\Omega$ is a Siegel domain and all convergent subsequence of $\left(f^{n}\right)$ converge to an automorphism of $\Omega$.

Proof. Since $\Omega$ is recurrent, there is $p_{0} \in \Omega$ and $\left(n_{i}\right)$ such that $f^{n_{1}}\left(p_{0}\right) \rightarrow p_{0}$. Taking a subsequence of $f^{n_{i+1}-n_{i}}$, we can assume that $f^{n_{i+1}-n_{i}}$ u.c.c. to a holomorphic map $h: \Omega \rightarrow \bar{\Omega}$. We have that $h(p)=p$. If for some $h$, rank $h=0$, then $h(\Omega)=p$ and $f(p)=p$. Consequently $p$ should be attracting and we are in case i). The last assertion is classical, see [RR] for example.

Assume, for some $h$, the rank of $h$ is two. Then if $a$ denotes the constant, jacobian determinant of $f$, we necessarily have $|a|=1$ and so $f$ is volume preserving. Let $G=\left\{f^{n}\right\}_{n \in \mathbf{Z}}$ where the closure is taken for the topology of u.c.c.. It follows from results of Cartan, see [N], that $G$ is a compact Lie group, isomorphic to $\mathbb{T}^{2} \times F$ or $\mathbb{T} \times F$ where $F$ is a finite commutative group. The argument is even simpler than the one given in the first paragraph since we have the information that $f$ is volume preserving.

We can assume that all limits $h$ have rank one and consequently $|a|<1$. Let $\Sigma=h(\Omega)$. We have $\Sigma \subset \bar{\Omega} \cap K$, so $\Sigma$ is a Riemann surface which is hyperbolic and $f: \Sigma \rightarrow \Sigma$ is an automorphism. Let $\Sigma_{p}$ be the irreducible component of $\Sigma \cap \Omega$ containing $p$. The difficulty here, as in Theorem 1.2, in order to say that $f$ is conjugate to a rotation on $\Sigma$ is that possibly other components of $\Sigma \cap \Omega$ might cluster on $\Sigma_{p}$. So the fact that say $\left(f^{n_{i}}\right)$ converge to identity on $\Sigma_{p}$ does not imply the convergence on $\Sigma$, considered as an 
abstract Riemann surface. As in Theorem 1.2 we show first that $f$ is not constant on any component $f^{l}\left(\Sigma_{p}\right), l \geqq 0$. Hence we can assume using a diagonal process that for a subsequence $\left(f^{m_{i}}\right), f^{m_{i}} \rightarrow h$ and $h=I d$ on $\bigcup_{l \geqq 0} f^{l}\left(\Sigma_{p}\right) \subset\{q \in \Omega ; h(q)=q\}$. Consequently $\bigcup_{l \geqq 0} f^{l}\left(\Sigma_{p}\right)$ is closed in $\Omega$. Since $f^{m_{t}} \rightarrow I d$ on $\Sigma_{p}$ and since $f$ is not of finite order, see [FM], then $f$ is conjugate to an irrational rotation on the Riemann surface $\Sigma$. We then show as in Theorem 2.3 of [FS3] that $\Sigma$ is contained in $\Omega$, so $\Sigma$ is closed in $\Omega$. The rest of the argument is as in Theorem 1.2 or as in [FS3] Theorem 2.3.

\section{References}

[B] Beardon, A.: Iteration of rational functions. Springer Verlag (1991)

[BBD] Barrett, D., Bedford, E., Dadok, J.: $T^{n}$-actions on holomorphically separable complex manifolds. Math. Z. 202 (1989), 65-82

[BS] Bedford, E., Smillie, J.: Polynomial diffeomorphisms of $\mathbb{C}^{2}$. II. Amer. J. Math. 4 (1991), 657-679

[CG] Carleson, L., Gamelin, T.: Complex dynamics. Springer Verlag (1993)

[FM] Friedland, S., Milnor, J.: Dynamical properties of plane automorphisms. Ergodic theory and dynamical systems 9 (1989), 67-99

[FS1] Fornæss, J.E., Sibony, N.: Complex dynamics in higher dimension. I. Asterisque 222 (1994), 201-231

[FS2] Fornæss, J.E., Sibony, N.: Complex dynamics in higher dimension. II. To appear in Ann. Math. Studies

[FS3] Fornæss, J.E., Sibony, N.: Complex Henon mappings in ${ }^{2}$ and Fatou-Bieberbach domains. Duke Math. J. 65 (1992), 345-380

[FS4] Fornæss, J.E., Sibony, N.: Holomorphic dynamical systems. (To appear)

[G] Gavosto, E.: To appear

[H] Herman, M.: Recent results on some open questions on Siegel's linearization

[Ko] Kobayashi, S.: Hyperbolic manifolds and holomorphic mappings. New York, Marcel Dekker, 1970

[Kr] Kruzhilin, N.G.: Holomorphic automorphisms of hyperbolic Reinhardt domains. Math. USSR Izvestya 32 (1989), 15-38

[M] Milnor, J.: Dynamics in one complex variable: Introductory Lectures. SUNY Stony Brook. Institute for Mathematical Sciences. Preprint \#1990/5

[N] Narashiman, R.: Several complex variables. Univ. Chicago Press, 1971

[RR] Rosay, J.-P., Rudin, W.: Holomorphic maps from $\mathbb{C}^{n}$ to $\mathbb{C}^{n}$. TAMS 310 (1988), 47-86

[U] UEDA, T.: Fatou set in complex dynamics in projective spaces. Preprint 